\title{
Effect of Leading-Edge Pressure in the Boundary Layer under High Freestream Disturbances
}

\author{
Yasar Arafath Udhuman, Alakesh Chandra Mandal \\ Indian Institute of Technology Kanpur \\ Kanpur, Utter Pradesh,India - 208016 \\ yasaru@iitk.ac.in; alakeshm@iitk.ac.in
}

\section{Extended Abstract}

Some initial observations from our ongoing study on the effect of leading-edge geometry and the associated pressure gradient on a flat plate boundary layer under the high level of freestream disturbances are reported here. Measurements were carried out in a low-speed wind tunnel using the particle image velocimetry (PIV) and the hotwire anemometry techniques. Freestream turbulence (FST) was generated by placing a turbulent generating grid at the entrance of the tunnel test section. Two flat plates with the same thickness but with different leading edge (LE) geometries, i.e. an asymmetrically modified super-elliptic (AMSE) leading edge and a super-elliptic (SE) leading edge were used. Trailing-edge flap was used to alter the pressure distribution around the leading edges. Experiments were carried out for two different freestream cases, i.e. without and with a contoured wall at the top of the test section above the plate. To refer in the text, we call the flow to be zero pressure gradient (ZPG) flow in the absence of the contoured wall inside the test section.

Under ZPG flow, pressure distributions around the leading edges for flap deflections at $0^{\circ}$ and $4^{\circ}$ were found from the velocity measurements in the freestream; anti-clockwise flap deflection with respect to the streamwise direction is considered to be positive. For a low aspect ratio leading edge, SE, a strong pressure suction peak is found to exist near the leading edge for $0^{\circ}$ flap deflection, whereas the suction peak value is considerably reduced for $4^{\circ}$ flap deflection. The streamwise extent of the non-zero pressure gradient region over the working surface of the plate also reduces compared to $0^{\circ}$ flap deflection. As AMSE leading edge is an aerodynamically optimized configuration [1], no appreciable pressure suction peak is observed even without flap deflection.

Measurements in the spanwise plane of the boundary layer under the high level of FST were conducted using the timeresolved PIV (TR-PIV) technique. The results show high- and low-speed streaks inside the boundary layer for the AMSE leading edge at $0^{\circ}$ flap deflection, consistent with previous studies [e.g. 2-4]. However, by changing the leading edge geometry to SE, we find that the flow is populated with lambda-shaped structures instead of high- and low-speed streaks. Similar structures are also reported for wake-induced boundary layer transition [5,6]. For this leading edge at $0^{\circ}$ flap deflection, a strong suction peak is found to occur around the leading edge which leads to these lambda-shaped structures. However, the positive flap deflection for this leading edge eliminates the suction peak around the leading edge which results in the disappearance of these structures leading to the dominance of streaky structures.

In order to confirm that the sharp pressure drop near leading edge plays a key role in the generation of such structures, an adverse pressure gradient is intentionally imposed on the flow using a contoured wall at the top in the test section. Measurements in the spanwise plane of the flat plate with AMSE leading edge are then carried out under this condition. The flow which was populated with streaky structures without the contoured wall at $0^{\circ}$ flap deflection, is found to be populated again similar lambda-shaped structures with the presence of the contoured wall at $0^{\circ}$ flap deflection. However, the positive flap deflection again eliminates these structures and the flow is dominated by the streaky structures. These observations clearly indicate that the pressure distribution around the leading edge determines the development of flow structures downstream of the leading edge of a flat plate.

\section{References}

[1] Ronald E. Hanson, Howard P. Buckley, and Philippe Lavoie, "Aerodynamic optimization of the flat-plate leading edge for experimental studies of laminar and transitional boundary layers," Experiments in Fluids, vol. 53, no. 4, pp. 863871, 2012. 
[2] M. Matsubara, and P. Henrik Alfredsson, "Disturbance growth in boundary layers subjected to free-stream turbulence," Journal of Fluid Mechanics, vol. 430, pp. 149-168, 2001.

[3] A. C. Mandal, L. Venkatakrishnan, and J. Dey, "A study on boundary-layer transition induced by free-stream turbulence," Journal of Fluid Mechanics, vol. 660, pp. 114-146, 2010.

[4] G. Balamurugan and A. C. Mandal, "Experiments on localized secondary instability in bypass boundary layer transition," Journal of Fluid Mechanics, vol. 817, pp. 217-263, 2017.

[5] C. Pan, J. J. Wang, P. F. Zhang \& L. H. Feng, "Coherent structures in bypass transition induced by a cylinder wake," Journal of Fluid Mechanics, vol. 603, pp. 367-389, 2008.

[6] A. C. Mandal, J. Dey, "An experimental study of boundary layer transition induced by a cylinder wake," Journal of Fluid Mechanics, vol. 684, pp. 60-84, 2011. 